\title{
Quadrupole moment of the nucleon in chiral constituent quark model
}

\author{
Harleen Dahiya* ${ }^{\dagger}$ \\ Dr. B.R. Ambedkar National Institute of Technology, Jalandhar 144011 INDIA \\ E-mail: harleen_dahiya@yahoo.com

\section{Neetika Sharma} \\ Dr. B.R. Ambedkar National Institute of Technology, Jalandhar 144011 INDIA \\ E-mail: neetikaphy@gmail.com
}

\begin{abstract}
The electromagnetic form factors are the most fundamental quantities to describe the internal structure of the nucleon and the shape of a spatially extended particle is determined by its intrinsic quadrupole moment which is first order moment of the charge density operator. With some experimental indications of a deformed nucleon, we have calculated the intrinsic quadrupole moment of the octet and decuplet baryons in the framework of chiral constituent quark model $\chi \mathrm{CQM}$ which is quite successful in explaining some of the important baryon properties in the nonperturbative regime.
\end{abstract}

Light Cone 2010: Relativistic Hadronic and Particle Physics

June 14-18, 2010

Valencia, Spain

\footnotetext{
${ }^{*}$ Speaker.

${ }^{\dagger}$ H.D. would like to thank the organizers of Light Cone 2010 and Department of Science and Technology, Government of India, for financial support.
} 


\section{Introduction}

The electromagnetic form factors are the fundamental quantities of theoretical and experimental interest to investigate the internal structure of nucleon. The knowledge of internal structure of nucleon in terms of quarks and gluons degrees of freedom of Quantum Chromodynamics (QCD) provide a basis for understanding more complex, strongly interacting matter. While QCD is accepted as the fundamental theory of strong interactions, it cannot be solved accurately in the nonperturbative regime. A coherent understanding of the hadron structure in this energy regime is necessary to describe the strong interactions as they are sensitive to the pion cloud and provide a test for the QCD inspired effective field theories based on the chiral symmetry. Recently, a wide variety of accurately measured data have been accumulated for the static properties of baryons, for example, masses, electromagnetic moments, charge radii, and low energy dynamical properties such as scattering lengths and decay rates etc.. The charge radii and quadrupole moments are important observables in hadronic physics as they lie in the nonperturbative regime of QCD and give valuable information on the internal structure of hadrons.

The Naive Quark Model (NQM) is unable to explain the magnitude and sign of deformation measured for the case of nucleon and $\Delta^{+}$. A promising approach offered to solve QCD in this energy regime are the constituent quark models which can be constructed so as to include the relevant properties of QCD coming from the consequences of the spontaneous breaking of chiral symmetry $(\chi \mathrm{SB})$. One of the important nonperturbative approaches in this energy regime is chiral constituent quark model $(\chi \mathrm{CQM})[1]$. The $\chi \mathrm{CQM}$ coupled with the "quark sea" generation through the chiral fluctuation of a constituent quark into a Goldstone bosons (GBs) [2, 3, 4], successfully explains the "proton spin crisis" [5], hyperon $\beta$ decay parameters [6], the octet and decuplet baryons magnetic moments [7]. The extension to the SU(4) symmetry successfully predicts the contribution of intrinsic charm (IC) content in the low lying and charmed baryon magnetic moments and their radiative decay widths [8]. In this context, it become desirable to extend the model to other low energy properties like charge radii, quadrupole moment, and higher order moments of the multipole expansion.

The purpose of present communication is to calculate the intrinsic quadrupole moment of the octet and decuplet baryons within the framework of $\chi \mathrm{CQM}$ using the general parameterization method. In order to understand the important role played by the pion cloud and SU(3) symmetry breaking in measuring the quadrupole moment we would carry out the calculations with and without symmetry breaking. The results have also been compared with the NQM predictions and the latest available data. Further, it would also be interesting to understand in detail the role of GP model parameters in the determination in of baryon quadrupole moment.

\section{Intrinsic quadrupole moment of the nucleon}

The mean square charge radii $\left(r_{B}^{2}\right)$ and quadrupole moments $\left(Q_{B}\right)$ are the lowest order moments of the charge density operator $\rho$ in a low-momentum expansion. For example, for any baryon $|B\rangle$ with charge $e_{B}$, the terms up to order of $q^{2}$ for the charge density are

$$
\langle\mathrm{B}|\rho(q)| B\rangle=e_{B}-\frac{q^{2}}{6} r_{B}^{2}-\frac{q^{2}}{6} Q_{B}+\ldots \ldots,
$$


where $q$ is the photon momentum transferred to the baryon. The first two terms arise due to the spherically symmetric monopole part of charge density, while the third term arises due to the quadrupole part of the charge density. They characterize the total charge, spatial extension of charge radii, and shape of the system, respectively.

The shape of a spatially extended particle is determined by its quadrupole moment $[9,10,11]$, corresponding to the charge quadrupole form factor $G_{C 2}\left(q^{2}\right)$ at zero momentum transfer. The intrinsic quadrupole moment of a nucleus with respect to the body frame of axis is defined as

$$
Q_{0}=\int d^{3} r \rho(\mathbf{r})\left(3 z^{2}-r^{2}\right)
$$

If the charge density is concentrated along the $z$-direction (symmetry axis of the particle), the term proportional to $3 z^{2}$ dominates, $Q_{0}$ is positive, and the particle is prolate shaped. If the charge density is concentrated in the equatorial plane perpendicular to $z$ axis, the term proportional to $r^{2}$ prevails, $Q_{0}$ is negative and the particle is oblate in shape. The angular momentum selection rule however, does not allow the spin $\frac{1}{2}^{+}$baryons to have any spectroscopic quadrupole moment. Therefore, the $\gamma^{*} N_{\frac{1}{2}}{ }^{+} \rightarrow \Delta_{\frac{3}{2}}^{+}$transition is studied to understand the quadrupole amplitudes in the nucleon as well as $\Delta^{+}$.

The spin and parity conservation in the $\Delta^{+} p$ transition require three contributing amplitudes, magnetic dipole $M 1$, the electric quadrupole moment $E 2$, and the charge quadrupole moment $C 2$ photon absorption amplitudes. The information on the intrinsic quadrupole moments can be obtained from the measurements of $E 2$ and $C 2$ amplitudes [12,13]. If the charge distribution of the initial and final three-quark states were spherically symmetric, the $E 2$ and $C 2$ amplitudes of the multipole expansion would be zero [14]. However, the recent experiments at JLAB, SELEX Collaboration reveal that although these quadrupole amplitudes are small compared to the dominant magnetic dipole transition $M 1$, they are clearly non zero [15]. More recently, the quadrupole transition moment $\left(Q_{\Delta^{+} N}\right)$ measured by LEGS and Mainz Collaborations is $-0.108 \pm 0.009 \pm 0.034 \mathrm{fm}^{2}$ [16] and $-0.0846 \pm 0.0033 \mathrm{fm}^{2}$ [12], respectively. These measurements lead to the conclusion that the nucleon and the $\Delta^{+}$are intrinsically deformed.

\section{General Parameterization method}

In order to predict the sign as well as magnitude of deformation in the octet and decuplet baryons, we have used the general parameterization (GP) method [17]. The charge quadrupole operator composed of a two- and three-body operator terms in spin-flavor space is given as

$$
Q=\mathrm{B} \sum_{i \neq j}^{3} e_{i}\left(3 \sigma_{i z} \sigma_{j z}-\sigma_{\mathbf{i}} \cdot \sigma_{\mathbf{j}}\right)+\mathrm{C} \sum_{i \neq j \neq k}^{3} e_{i}\left(3 \sigma_{j z} \sigma_{k z}-\sigma_{\mathbf{j}} \cdot \sigma_{\mathbf{k}}\right),
$$

where the coefficients $\mathrm{B}$ and $\mathrm{C}$ are the constants to be determined from the experimental observations on charge radii and quadrupole moments. The quadrupole moments $Q$ for the octet and decuplet baryons can be calculated from Eq. (3.1) by evaluating matrix elements of the operator corresponding to the three-quark spin-flavor wave functions $\left(Q_{B}=\langle B|Q| B\rangle\right)$. It is straightforward to verify that

$$
\sum_{i \neq j} e_{i}\left(\sigma_{i} \cdot \sigma_{j}\right)=2 J \cdot \sum_{i} e_{i} \sigma_{i}-3 \sum_{i} e_{i}
$$




$$
\sum_{i \neq j \neq k} e_{i}\left(\sigma_{j} \cdot \sigma_{k}\right)= \pm 3 \sum_{i} e_{i}-\sum_{i \neq j} e_{i}\left(\sigma_{i} \cdot \sigma_{j}\right)
$$

In Eq. (3.3), + sign holds for $J=\frac{3}{2}$ and - sign for $J=\frac{1}{2}$ states. Using the expectation value of operator $2 J \cdot \sum_{i} e_{i} \sigma_{i}$ between the baryon wavefunctions $|B\rangle$ in the initial and final states [18], the operators in Eqs. (3.2) and (3.3) become

$$
\begin{array}{c|c|c}
\text { Operator } & \sum_{i \neq j} e_{i}\left(\sigma_{i} \cdot \sigma_{j}\right) & \sum_{i \neq j \neq k} e_{i}\left(\sigma_{j} \cdot \sigma_{k}\right) \\
\hline J=\frac{1}{2} & 3 \sum_{i} e_{i} \sigma_{i z}-3 \sum_{i} e_{i} & -3 \sum_{i} e_{i} \sigma_{i z} \\
J=\frac{3}{2} & 5 \sum_{i} e_{i} \sigma_{i z}-3 \sum_{i} e_{i} & 6 \sum_{i} e_{i}-5 \sum_{i} e_{i} \sigma_{i z}
\end{array}
$$

The expression for the quadrupole moment of the octet and decuplet baryons in Eq. (3.1) can be expressed as

$$
\begin{aligned}
Q_{1 / 2} & =3 \mathrm{~B} \sum_{i \neq j} e_{i} \sigma_{\mathbf{i z}} \sigma_{\mathbf{j} \mathbf{z}}+3 \mathrm{C} \sum_{\mathbf{i} \neq \mathbf{j} \neq \mathbf{k}} \mathbf{e}_{\mathbf{i}} \sigma_{\mathbf{j} \mathbf{z}} \sigma_{\mathbf{k z}}+(-\mathbf{3 B}+\mathbf{3 C}) \sum_{\mathbf{i}} \mathbf{e}_{\mathbf{i}} \sigma_{\mathbf{i z}}+3 \mathrm{~B} \sum_{\mathbf{i}} \mathbf{e}_{\mathbf{i}}, \\
Q_{3 / 2} & =3 \mathrm{~B} \sum_{i \neq j} e_{i} \sigma_{\mathbf{i z}} \sigma_{\mathbf{j} \mathbf{z}}+3 \mathrm{C} \sum_{\mathbf{i} \neq \mathbf{j} \neq \mathbf{k}} \mathbf{e}_{\mathbf{i}} \sigma_{\mathbf{j} \mathbf{z}} \sigma_{\mathbf{k z}}+(-\mathbf{5 B}+\mathbf{5 C}) \sum_{\mathbf{i}} \mathbf{e}_{\mathbf{i}} \sigma_{\mathbf{i z}}+(3 \mathrm{~B}-\mathbf{6 C}) \sum_{\mathbf{i}} \mathbf{e}_{\mathbf{i}},
\end{aligned}
$$

where $i=(u, d, s)$ for any of the three quarks. Therefore, the calculation of quadrupole moment reduces to the calculation of the flavor structure $\left(\sum_{i} e_{i}\right)$, spin structure $\left(\sum_{i} e_{i} \sigma_{i z}\right)$ and the tensor terms $\left(\sum_{i} e_{i} \sigma_{i z} \sigma_{j z}\right.$ and $\left.\sum_{i} e_{i} \sigma_{j z} \sigma_{k z}\right)$ for a given baryon.

The spin and flavor structure of a given baryon can be calculated using the SU(6) spin-flavor symmetry of the wave functions. The expectation value of the appropriate operators are expressed as $\widehat{e_{i}} \equiv\left\langle B\left|\sum_{i} e_{i}\right| B\right\rangle$, and $\widehat{e_{i} \sigma_{i z}} \equiv\left\langle B\left|\sum_{i} e_{i} \sigma_{i z}\right| B\right\rangle$, where $|B\rangle$ is the baryon wave function and $e_{i}$ and $\sigma_{i}$ are the charge and spin operators defined as

$$
\begin{aligned}
\sum_{i} e_{i} & =\sum_{q=u, d, s} n_{q}^{B} q+\sum_{\bar{q}=\bar{u}, \bar{d}, \bar{s}} n_{\bar{q}}^{B} \bar{q}=n_{u}^{B} u+n_{d}^{B} d+n_{s}^{B} s+n_{\bar{u}}^{B} \bar{u}+n_{d}^{B} \bar{d}+n_{s}^{\underline{B}} \bar{s} \\
\sum_{i} e_{i} \sigma_{i z} & =\sum_{q=u, d, s}\left(n_{q_{+}}^{B} q_{+}+n_{q_{-}}^{B} q_{-}\right)=n_{u_{+}}^{B} u_{+}+n_{u_{-}}^{B} u_{-}+n_{d_{+}}^{B} d_{+}+n_{d_{-}}^{B} d_{-}+n_{s_{+}}^{B} s_{+}+n_{s_{-}}^{B} s_{-} .
\end{aligned}
$$

Here $n_{q}^{B}\left(n_{q}^{\underline{B}}\right)$ is the number of quarks with charge $q(\bar{q})$, and $n_{q_{+}}^{B}\left(n_{q_{-}}^{B}\right)$ is the number of $q$ quarks with spin $+(-)$. The tensor terms can be simplified and further reduced to the calculations of spin and flavor structure as presented in Eq. (3.4).

For the case of octet baryons, Eq. (3.5) can be solved for the spin-flavor symmetric SU(6) octet baryon wave function by using the operators defined in Eqs. (3.7) and (3.8). For ready reference, the quadrupole moment of proton, neutron and $\Sigma^{+}$are expressed as

$$
\begin{aligned}
Q_{p} & =3 \mathrm{~B}\left(-2 u_{+}-d_{+}+2 u+d\right)+\mathrm{C}\left(-4 u+d+4 u_{+}-d_{+}\right), \\
Q_{n} & =3 \mathrm{~B}\left(-u_{+}-2 d_{+}+u+2 d\right)+\mathrm{C}\left(u-4 d-u_{+}+4 d_{+}\right), \\
Q_{\Sigma^{+}} & =3 \mathrm{~B}\left(-2 u_{+}-s_{+}+2 u+s\right)+\mathrm{C}\left(-4 u+s+4 u_{+}-s_{+}\right) .
\end{aligned}
$$

Similarly, the quadrupole moment of the decuplet baryon $\Delta^{+}$can be calculated from Eq. (3.6) by solving the matrix elements corresponding to the decuplet baryon wave function and by using the operators defined in Eqs. (3.7) and (3.8). We have

$$
Q_{\Delta^{+}}=\mathrm{B}\left(2 u_{+}+d_{+}+3(2 u+d)\right)+\mathrm{C}\left(-3(2 u+d)+5\left(2 u_{+}+d_{+}\right)\right) .
$$


In the case of naive quark model (NQM), the calculations lead to $u=\frac{2}{3}, d=-\frac{1}{3}, s=-\frac{1}{3}$, $u_{+}=\frac{2}{3}, d_{+}=-\frac{1}{3}$, and $s_{+}=-\frac{1}{3}$. The quadrupole moments of $p, n, \Sigma^{+}$, and $\Delta^{+}$are now given as

$$
Q_{p}=0, \quad Q_{n}=0, \quad Q_{\Sigma^{+}}=0, \quad Q_{\Delta^{+}}=4 \mathrm{~B}+2 \mathrm{C} .
$$

These results suggest that the octet baryons are spherically symmetric and there is no deformation. However, the experimental results suggest that the nucleon is intrinsically deformed. Therefore, there is a need to go beyond this model to include the effects of "quark sea".

\section{Chiral constituent quark model}

The basic process in the $\chi \mathrm{CQM}$ is the emission of a GB by a constituent quark which further splits into a $q \bar{q}$ pair as $q_{ \pm} \rightarrow \mathrm{GB}^{0}+q_{\mp}^{\prime} \rightarrow(q \vec{q})+q_{\mp}^{\prime}$, where $q \vec{q}+q^{\prime}$ constitute the "quark sea" $[2,3,4]$. The effective Lagrangian describing interaction between quarks and a nonet of GBs is $\mathscr{L}=g_{8} \bar{q} \Phi^{\prime} q$, where $g_{8}$ is the coupling constant for the GBs. The GB field $\Phi^{\prime}$ is expressed in terms of the quark contents as

$$
\Phi^{\prime}=\left(\begin{array}{ccc}
\phi_{u u} u \bar{u}+\phi_{u d} d \bar{d}+\phi_{u s} s \bar{s} & \varphi_{u d} u \bar{d} & \varphi_{u s} u \bar{s} \\
\varphi_{d u} d \bar{u} & \phi_{d u} u \bar{u}+\phi_{d d} d \bar{d}+\phi_{d s} s \bar{s} & \varphi_{d s} d \bar{s} \\
\varphi_{s u} s \bar{u} & \phi_{s d} s \bar{d} & \phi_{s u} u \bar{u}+\phi_{s d} d \bar{d}+\phi_{s s} s \bar{s}
\end{array}\right)
$$

where

$$
\begin{aligned}
& \phi_{u u}=\phi_{d d}=\frac{1}{2}+\frac{\beta}{6}+\frac{\zeta}{3}, \quad \phi_{s s}=\frac{2 \beta}{3}+\frac{\zeta}{3}, \quad \phi_{u s}=\phi_{d s}=\phi_{s u}=\phi_{s d}=-\frac{\beta}{3}+\frac{\zeta}{3}, \\
& \phi_{d u}=\phi_{u d}=-\frac{1}{2}+\frac{\beta}{6}+\frac{\zeta}{3}, \quad \varphi_{u d}=\varphi_{d u}=1, \quad \varphi_{u s}=\varphi_{d s}=\varphi_{s u}=\varphi_{s d}=\alpha .
\end{aligned}
$$

SU(3) symmetry breaking is introduced by considering $M_{S}>M_{u, d}$ as well as by considering the masses of GBs to be nondegenerate $\left(M_{K, \eta}>M_{\pi}\right.$ and $\left.M_{\eta^{\prime}}>M_{K, \eta}\right)[2,3,4,5]$. The parameter $a(=$ $\left.\left|g_{8}\right|^{2}\right)$ denotes the transition probability of chiral fluctuation of the splitting $u(d) \rightarrow d(u)+\pi^{+(-)}$, whereas $\alpha^{2} a, \beta^{2} a$ and $\zeta^{2} a$ respectively, denote the probabilities of transitions of $u(d) \rightarrow s+K^{-(o)}$, $u(d, s) \rightarrow u(d, s)+\eta$, and $u(d, s) \rightarrow u(d, s)+\eta^{\prime}$.

Since the quadrupole moment operators for the spin $\frac{1}{2}$ and spin $\frac{3}{2}$ baryons involve the knowledge of spin and flavor structure of baryons, it is important to mention here that redistribution of flavor and spin take place among the "quark sea". The modified flavor and spin structure of the baryon in $\chi \mathrm{CQM}$ due to the chiral symmetry breaking can be calculated by substituting for every constituent quark

$$
q \rightarrow P_{q} q+|\psi(q)|^{2} \quad \text { and } \quad q_{ \pm} \rightarrow P_{q} q_{ \pm}+\left|\psi\left(q_{ \pm}\right)\right|^{2}
$$

where $P_{q}=1-\sum P_{q}$ is the transition probability of no emission of GB from any of the $q$ quark, $|\psi(q)|^{2}$ is the transition probability of the $q$ quark, and $\left|\psi\left(q_{ \pm}\right)\right|^{2}$ is the probability of transforming a $q_{ \pm}$quark [5]. 


\section{Results and Discussion}

In order to calculate the quadrupole moments of the octet and decuplet baryons in the $\chi \mathrm{CQM}$, we substitute Eq. (4.3) in Eqs. (3.5) and (3.6) . For example, on substituting Eq. (4.3) in Eqs. (3.9), (3.10) and (3.11), the quadrupole moments of $p, n$, and $\Sigma^{+}$in $\chi \mathrm{CQM}$ can now be expressed as

$$
\begin{aligned}
Q_{p} & =\mathrm{B} a\left(6+\beta^{2}+2 \zeta^{2}\right)-\mathrm{C} a\left(4+2 \alpha^{2}+\beta^{2}+2 \zeta^{2}\right), \\
Q_{n} & =\mathrm{B} a\left(3-3 \alpha^{2}\right)+\mathrm{C} \frac{a}{3}\left(3+9 \alpha^{2}+2 \beta^{2}+4 \zeta^{2}\right), \\
Q_{\Sigma^{+}} & =\mathrm{B} a\left(6+\alpha^{2}+2 \zeta^{2}\right)-\mathrm{C} \frac{a}{3}\left(5 \alpha^{2}+4 \beta^{2}+12+6 \zeta^{2}\right) .
\end{aligned}
$$

From the above equations, we can directly estimate the effects of SU(3) symmetry breaking and pion cloud in the quadrupole moments of the octet baryons. It is clear from the equations that, for the non zero value of the GP parameters (B and $\mathrm{C}$ ), there is a significant contribution of the $\chi \mathrm{CQM}$ parameters $(a, \alpha, \beta, \zeta)$ to the quadrupole moments. Similarly, the quadrupole moment of $\Delta^{+}$and $\Xi^{* 0}$ in $\chi \mathrm{CQM}$, after substituting the contribution coming from the "quark sea", can be expressed as

$$
\begin{aligned}
& Q_{\Delta^{+}}=\mathrm{B}\left(4-\frac{1}{3} a\left(6+\beta^{2}+2 \zeta^{2}\right)\right)+\mathrm{C}\left(2-\frac{5}{3} a\left(6+\beta^{2}+2 \zeta^{2}\right)\right), \\
& Q_{\Xi^{* 0}}=\mathrm{B} \frac{a}{3}\left(-3+\alpha^{2}+2 \beta^{2}\right)+\mathrm{C} \frac{5 a}{3}\left(-3+\alpha^{2}+2 \beta^{2}\right) .
\end{aligned}
$$

Before giving the numerical results, we would like to discuss the input parameters involved in the calculations of baryon quadrupole moments in $\chi \mathrm{CQM}$. The calculations involve the two set of parameters, the ones corresponding to the GP method (B and C, where $\mathrm{B}>\mathrm{C}$ ), and other corresponding to the $\chi \mathrm{CQM}$ symmetry breaking $(a, \alpha, \beta$, and $\zeta$ ). In order to fix the values of GP parameters, we have performed a fit to the available experimental values of the baryon charge radii leading to $\mathrm{B}=-0.0525, \mathrm{C}=-0.0158$, as the best fit. For the $\chi \mathrm{CQM}$ parameters, we have used the same set of parameters as discussed in our earlier publication [6]. The values used are $a=0.12, \quad \alpha=0.45, \quad \beta=0.45, \quad \zeta=-0.15$.

Using the above discussed set of parameters, we have calculated the quadrupole moments of octet and decuplet baryons in $\chi \mathrm{CQM}$ and results have been presented in Tables 1 and 2. In order to understand the role of chiral symmetry breaking and $\mathrm{SU}(3)$ symmetry breaking, we have also presented the results of NQM in the tables. Most of the models in literature are unable to estimate the intrinsic quadrupole moment of the baryons. In the case of octet baryons there are indirect evidences of small deformation in the nucleon. This deformation can easily be observed in our results. For the case of decuplet baryons also, $\chi \mathrm{CQM}$ is able to give a fairly good prediction of the sign as well as magnitude of the quadrupole moments.

In order to understand the effect of three-quark contributing term and to make our calculations more responsive, we have also presented the results by neglecting this contribution which can be obtained by fixing the coefficient $\mathrm{C}=0$. From Table 1 , it is clear that the results are affected to a very small extent by the inclusion of three-quark term. The three-quark terms do not seem to play any significant role in the case of octet baryons. However, in the case of decuplet baryons, the 


\begin{tabular}{|c|c|c|}
\hline Baryon & \multicolumn{2}{|c|}{$\chi \mathrm{CQM}$} \\
\hline & $\mathrm{C}=0$ & $\mathrm{C}=-0.016$ \\
\hline$Q_{p}$ & -0.041 & -0.032 \\
$Q_{n}$ & -0.016 & -0.019 \\
$Q_{\Sigma^{+}}$ & -0.041 & -0.032 \\
$Q_{\Sigma^{-}}$ & 0.010 & 0.009 \\
$Q_{\Sigma^{0}}$ & -0.016 & -0.012 \\
$Q_{\Xi^{0}}$ & -0.016 & -0.019 \\
$Q_{\Xi^{-}}$ & 0.010 & 0.009 \\
\hline
\end{tabular}

Table 1: Quadrupole moments of spin $\frac{1}{2}^{+}$baryons in $\chi \mathrm{CQM}$. The deformation is zero for all the cases in NQM.

\begin{tabular}{|c|c|c|c|}
\hline Baryon & NQM & \multicolumn{2}{|c|}{$\chi \mathrm{CQM}$} \\
\hline & & $\mathrm{C}=0$ & $\mathrm{C}=-0.016$ \\
\hline$Q_{\Delta^{++}}$ & -0.484 & -0.398 & -0.428 \\
$Q_{\Delta^{+}}$ & -0.242 & -0.196 & -0.208 \\
$Q_{\Delta^{0}}$ & 0.0 & 0.005 & 0.013 \\
$Q_{\Delta^{-}}$ & 0.242 & 0.207 & 0.208 \\
$Q_{\Sigma^{*+}}$ & -0.242 & -0.196 & -0.208 \\
$Q_{\Sigma^{*-}}$ & 0.242 & 0.207 & 0.234 \\
$Q_{\Sigma^{* 0}}$ & 0.0 & 0.005 & 0.013 \\
$Q_{\Xi^{* 0}}$ & 0.0 & 0.005 & 0.013 \\
$Q_{\Xi^{*-}}$ & 0.242 & 0.207 & 0.234 \\
$Q_{\Omega^{-}}$ & 0.315 & 0.296 & 0.296 \\
\hline
\end{tabular}

Table 2: Quadrupole moments of spin $\frac{3}{2}^{+}$baryons in $\chi \mathrm{CQM}$.

results in Table 2 reveal that the inclusion of three-quark term increases the quadrupole moments thus making them significant in this case. It is interesting to observe that the effect of three-quark contribution is even more in the case of neutral baryons. For example, in the case of charged baryons, the predictions are increased about $10 \%$ whereas for the case of neutral particles the variation is more then $50 \%$ making the effect of three-quark term significant.

Since the electric quadrupole moment of the octet baryons as well as that of octet-decuplet transitions are amenable to measurement, any experimental information would have important implications for the basic tenets of $\chi \mathrm{CQM}$ and the effects of SU(3) symmetry breaking.

\section{References}

[1] S. Weinberg, Physica A 96, 327 (1979); A. Manohar and H. Georgi, Nucl. Phys. B 234, 189 (1984).

[2] T.P. Cheng and Ling Fong Li, Phys. Rev. Lett. 74, 2872 (1995); Phys. Rev. D 57, 344 (1998).

[3] J. Linde, T. Ohlsson, and H. Snellman, Phys. Rev. D 57, 452 (1998); 57, 5916 (1998). 
[4] X. Song, J.S. McCarthy, and H.J. Weber, Phys. Rev. D 55, 2624 (1997); X. Song, Phys. Rev. D 57, 4114 (1998).

[5] H. Dahiya and M. Gupta, Phys. Rev. D 64, 014013 (2001); Int. J. Mod. Phys. A 19, 5027 (2004); Int. J. Mod. Phys. A 21, 4255 (2006); Phys. Rev. D 78, 014001 (2008).

[6] N. Sharma, H. Dahiya, P.K. Chatley, and M. Gupta, Phys. Rev. D 79, 077503 (2009); N. Sharma, H. Dahiya, and P.K. Chatley Eur. Phys. J. A 44, 125 (2010).

[7] H. Dahiya and M. Gupta, Phys. Rev. D 66, 051501(R) (2002); 67, 114015 (2003).

[8] H. Dahiya and M. Gupta, Phys. Rev. D 67, 074001 (2003); N. Sharma, H. Dahiya, P.K. Chatley and M. Gupta, D 81, 073001 (2010).

[9] A.J. Buchmann and E. M. Henley, Phys. Rev. C 63, 015202 (2001).

[10] A.J. Buchmann, Phys. Rev. Lett. 93, 212301 (2004).

[11] A.J. Buchmann, AIP Conf. Proc. 904, 110 (2007).

[12] G. Blanpied et al. (The LEGS Collaboration), Phys. Rev. C 64, 025203 (2001); L. Tiator et al., Eur. Phys. J. A 17, 357 (2003).

[13] A.M. Bernstein, Eur. Phys. J. A 17, 349 (2003); C.N. Papanicolas, Eur. Phys. J. A 18, 141 (2003).

[14] C. Becchi and G. Morpurgo, Phys.Lett. 17, 352, (1965).

[15] C. Amsler et al. (Particle Data Group), Phys. Lett. B 667, 1 (2008).

[16] G. Blanpied et al., Phys. Rev. Lett. 79, 4337 (1997).

[17] G. Morpurgo, Phys. Rev. D 40, 2997 (1989); G. Dillon and G. Morpurgo, Phys. Lett. B 448, 107 (1999).

[18] A. Le Yaouanc et al., Hadron Transitions in the Quark Model, Gordon and Breach (1988). 